\title{
Article \\ Ischemic Etiology and Prognosis in Men and Women with Acute Heart Failure
}

\author{
Lourdes Vicent ${ }^{1}$ D, Jose Guerra ${ }^{2,3}{ }^{\mathbb{D}}$, Rafael Vazquez-García ${ }^{4}\left(\mathbb{D}\right.$, José R. Gonzalez-Juanatey ${ }^{3,5}$, \\ Luis Martínez Dolz ${ }^{6}{ }^{(D}$, Javier Segovia ${ }^{3,7}$ (D), Domingo Pascual-Figal ${ }^{8}\left(\mathbb{D}\right.$, Ramón Bover ${ }^{9}$, Fernando Worner ${ }^{10}$,

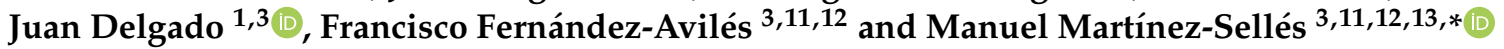

1 Cardiology Department, Hospital Universitario 12 de Octubre, 28028 Madrid, Spain; mlourdesvicent@gmail.com (L.V.); juan.delgado@salud.madrid.org (J.D.)

2 Cardiology Department, Hospital de la Santa Creu i Sant Pau, 08041 Barcelona, Spain; JGuerra@santpau.cat

3 CIBER de Enfermedades Cardiovasculares (CIBERCV), Instituto de Salud Carlos III, 28029 Madrid, Spain; Jose.Ramon.Gonzalez.Juanatey@sergas.es (J.R.G.-J.); jsecu@telefonica.net (J.S.); faviles@secardiologia.es (F.F.-A.)

4 Cardiology Department, Puerta del Mar University Hospital, 11009 Cádiz, Spain; rafael.vazquez.sspa@juntadeandalucia.es

5 Cardiology Department, University Hospital, 15076 Santiago de Compostela, Spain

6 Cardiology Department, University Hospital La Fe (CIBERCV), 46026 Valencia, Spain; luismartinezdolz@gmail.com

7 Cardiology Department, Hospital Universitario Puerta de Hierro Majadahonda, 28222 Madrid, Spain

8 Cardiology Department, Hospital Virgen de la Arrixaca, Department of Medicine, University of Murcia, 30120 Murcia, Spain; dpascual@um.es

check for updates

Citation: Vicent, L.; Guerra, J.; Vazquez-García, R.; Gonzalez-Juanatey, J.R.; Dolz, L.M.; Segovia, J.; Pascual-Figal, D.; Bover, R.; Worner, F.; Delgado, J.; et al. Ischemic Etiology and Prognosis in Men and Women with Acute Heart Failure. J. Clin. Med. 2021, 10, 1713. https://doi.org/10.3390/jcm10081713

Academic Editor: Ali Ahmed

Received: 21 March 2021

Accepted: 13 April 2021

Published: 15 April 2021

Publisher's Note: MDPI stays neutral with regard to jurisdictional claims in published maps and institutional affiliations.
9 Cardiology Department, Hospital Clínico San Carlos, 28040 Madrid, Spain; ramonboverfreire@gmail.com

10 Servicio de Cardiología, Hospital Universitari Arnau de Vilanova, Institut de Recerca Biomèdica de Lleida, 25198 Lleida, Spain; fworner.lleida.ics@gencat.cat

11 Cardiology Department, Instituto de Investigación, Hospital General Universitario Gregorio Marañón, 28007 Madrid, Spain

12 Facultad de Medicina, Universidad Complutense, 28040 Madrid, Spain

13 Facultad de Medicina, Universidad Europea, 28670 Madrid, Spain

* Correspondence: mmselles@secardiologia.es; Tel.: +34-915-8686-87

\begin{abstract}
Coronary heart disease is common in heart failure (HF). Our aim was to determine the impact of ischemic etiology on prognosis among men and women with HF. This study is a prospective national multicenter registry. The primary endpoint was 12-month mortality. Patients with HF and ischemic heart disease were stratified according to sex. A total of 1830 patients were enrolled of which $756(41.3 \%)$ were women. Ischemic etiology was more common in men (446 (41.6\%)) than in women $(167(22.2 \%))$. Among patients with ischemic HF, diabetes was more frequent in women than in men. Ischemic etiology was not associated with higher mortality risk, and this was true for women (Hazard Ratio [HR] 1.51, 95\% Confidence Interval [CI] 0.98-2.32; $p=0.61$ ) and men (HR 1.14, 95\% CI 0.81-1.61; $p=0.46)$, $p$-value for interaction: 0.067 . Mortality/readmission risk in ischemic HF increased in men with previous readmissions (HR 1.15, 95\% CI 1.02-1.29; $p=0.022$ ), chronic obstructive pulmonary disease (HR1.20, 95\% CI 1.02-1.41; $p=0.026$ ) and in women with diabetes (HR 2.23, 95\% CI 1.05-4.47; $p=0.035$ ). Ischemic etiology was not associated with mortality in HF patients. In ischemic HF, the variables associated with a poor prognosis were diabetes in women and previous readmissions and chronic obstructive pulmonary disease in men.
\end{abstract}

Keywords: heart failure; mortality; ischemic heart disease

\section{Introduction}

Ischemic heart disease is the leading cause of mortality worldwide and is a major cause of premature mortality and disability [1]. Ischemic heart disease is a common condition associated with heart failure (HF) and a powerful predisposing factor [2] of developing 
HF [3,4]. In addition, ischemic etiology has been associated with a poor prognosis in HF patients in some studies [5], but not in others [6].

Differences related to sex have been described in HF patients, including etiology, demographic profile, medical history, and prognosis [7-9]. Compared to men, women have a higher prevalence of hypertension, valvular heart disease, and diabetes but a lower prevalence of ischemic heart disease $[7,8,10]$. However, the effect of sex on the impact of ischemic heart disease on HF prognosis is controversial [11,12]. A worse prognosis has been described in women with coronary artery disease, with an increased risk of incident HF, cardiogenic shock [12], lower frequency of revascularization, and a lower use of antiplatelet drugs, statins, and beta-blockers $[10,13,14]$. Moreover, women have been underrepresented in clinical trials of $\mathrm{HF}$ and ischemic heart disease [15].

Our objective was to assess the impact of ischemic heart disease on HF prognosis in men and women. We have also compared the characteristics and treatment of women admitted with heart failure with their male counterparts.

\section{Methods}

The present study is a sub-analysis of the Spanish Network for the Study of Heart Failure II registry (REDINSCOR II). The methodology of the study has been previously detailed $[16,17]$. Briefly, the REDINSCOR II is a prospective, multicenter, nationwide study including adults admitted for acute HF in 20 Cardiology departments from Spanish hospitals, from October 2013 to December 2014. According to the definition of the current clinical practice guidelines, all patients had a diagnosis of acute HF at admission [18].

Patients with HF and ischemic heart disease were stratified according to sex. We considered ischemic etiology in the presence of coronary artery disease of sufficient severity and extension to justify myocardial damage $[19,20]$.

The primary endpoint was mortality at 12 months. We also analyzed the composite of all-cause mortality/hospital readmissions due to HF incidence of sudden cardiac death, mortality due to refractory HF, or heart transplantation at 12 months. Hospital readmissions and mortality at 1 and 6 months were also assessed.

This study was accomplished with the Declaration of Helsinki and was approved by the Ethics Committee of the recruiting hospitals (9/12/2013 CEIC: 57/2013; 19/09/2013 CEIC: 13/2013). All patients provided written informed consent.

\section{Statistical Analysis}

Continuous variables are shown as mean (standard deviation) or median (interquartile interval) for non-normally distributed variables. Categorical data are presented as frequencies and percentages. Continuous quantitative variables were compared using Student's $t$-test and ANOVA for the comparison of means or the Wilcoxon rank-sum in nonparametric data. Categorical variables were analyzed using the $\chi 2$ test and the Fischer exact test. Bonferroni's correction was applied for multiple comparisons.

Multivariate analysis included multiple logistic regression techniques and Cox regression modeling for the study endpoints. To determine which variables were entered into the final model, we used a sequential inclusion and exclusion method, with an inclusion $p$ threshold lower than 0.05 and exclusion over 0.1 . The final model included age, previous heart failure admissions, diabetes, glomerular filtration rate, HF therapies, rhythm, and anemia at discharge. All analyses were performed with the STATA software (StataCorp, College Station, TX, USA, version 14.0).

\section{Results}

A total of $1830 \mathrm{HF}$ patients were enrolled in the registry; the mean age was $73.3 \pm 10.6$ years, $756(41.3 \%)$ were women, and $613(33.5 \%)$ had ischemic etiology. Ischemic etiology was significantly more common in men (446 [41.5\%]) compared to women $(167(22.1 \%)), p<0.001$. Table 1 shows basal demographic characteristics in the studied patients according to sex and etiology. Compared to men, women were significantly older, and we found significant differences in 
the frequency of cardiovascular risk factors. Women with ischemic HF had a more common history of diabetes, while men more often had a history of tobacco and alcohol consumption. In patients with non-ischemic HF, the etiology of HF was valvular heart disease in 411 (33.9\%), hypertension in 535 (44.1\%), idiopathic/familiar in 199 (16.4\%), hypertrophic cardiomyopathy in $53(4.4 \%)$, and infiltrative diseases in $15(1.2 \%)$.

Table 1. Baseline characteristics in patients admitted with heart failure (HF) according to etiology and sex.

\begin{tabular}{|c|c|c|c|c|c|c|c|c|c|}
\hline & $\begin{array}{c}\text { Ischemic } \\
\text { HF }(n=613)\end{array}$ & $\begin{array}{c}\text { Non- } \\
\text { Ischemic HF } \\
(n=1218)\end{array}$ & $p$ & $\begin{array}{l}\text { Women, } \\
\text { Ischemic HF } \\
(n=167)\end{array}$ & $\begin{array}{c}\text { Men, } \\
\text { Ischemic } \\
\text { HF }(n=446)\end{array}$ & $p$ & $\begin{array}{c}\text { Women, } \\
\text { Non- } \\
\text { Ischemic } \\
\text { HF }(n=589)\end{array}$ & $\begin{array}{l}\text { Men, Non- } \\
\text { Ischemic } \\
\text { HF }(n=629)\end{array}$ & $p$ \\
\hline Age (years) & $73.3 \pm 10.6$ & $71.9 \pm 12.8$ & $<0.001$ & $77.2 \pm 10.3$ & $71.9 \pm 10.4$ & $<0.001$ & $74.7 \pm 11.7$ & $69.4 \pm 13.3$ & 0.002 \\
\hline $\begin{array}{c}\text { Previous heart failure } \\
\text { diagnosis }\end{array}$ & 415 (67.7) & $645(53.0)$ & $<0.001$ & $121(72.5)$ & $294(65.9)$ & 0.283 & $327(55.5)$ & $318(50.6)$ & 0.002 \\
\hline Years since the diagnosis & $3.0 \pm 5.3$ & $4.0 \pm 5.6$ & 0.019 & $3.4 \pm 4.9$ & $4.2 \pm 5.9$ & 0.107 & $3.3 \pm 5.8$ & $2.6 \pm 4.8$ & $<0.001$ \\
\hline $\begin{array}{c}\text { Previous heart failure } \\
\text { admissions }\end{array}$ & $290(47.3)$ & $455(37.3)$ & 0.003 & $84(50.3)$ & $206(46.2)$ & 0.366 & $220(37.4)$ & $235(37.4)$ & 0.997 \\
\hline $\begin{array}{l}\text { Tobacco use } \\
\text { Smoker } \\
\text { Former smoker }\end{array}$ & $\begin{array}{c}74(12.1) \\
269(44.0)\end{array}$ & $\begin{array}{c}361(29.7) \\
119(9.8)\end{array}$ & $<0.001$ & $\begin{array}{c}5(3.0) \\
17(10.2)\end{array}$ & $\begin{array}{c}69(15.5) \\
252(56.6)\end{array}$ & $\begin{array}{l}<0.001 \\
<0.001\end{array}$ & $\begin{array}{l}26(4.4) \\
54(9.2)\end{array}$ & $\begin{array}{l}307(49.1) \\
93(14.9)\end{array}$ & $\begin{array}{l}<0.001 \\
<0.001\end{array}$ \\
\hline Alcohol consumption & $54(8,8)$ & $139(11.4)$ & 0.002 & $1(0.6)$ & $53(11.9)$ & $<0.001$ & $10(1.7)$ & $129(20.5)$ & $<0.001$ \\
\hline Dyslipidemia & $429(70.0)$ & $583(47.9)$ & $<0.001$ & $109(65.3)$ & $320(71.8)$ & 0.192 & $303(51.4)$ & $280(44.6)$ & 0.044 \\
\hline Diabetes mellitus & $364(59.4)$ & $483(39.7)$ & $<0.001$ & $115(68.9)$ & $249(55.8)$ & 0.010 & $244(41.4)$ & $239(38.1)$ & 0.463 \\
\hline Hypertension & $540(88.1)$ & $874(71.8)$ & $<0.001$ & $150(89.8)$ & $390(87.4)$ & 0.547 & $444(75.4)$ & $430(68.5)$ & 0.025 \\
\hline Obesity & $282(46.0)$ & $609(50.0)$ & 0.106 & $78(46.7)$ & $204(45.7)$ & 0.831 & $299(50.8)$ & $310(49.3)$ & 0.606 \\
\hline $\begin{array}{l}\text { Chronic kidney disease } \\
-\quad \text { GFR }<30 \\
-\quad \text { GFR 30-59 }\end{array}$ & $\begin{array}{c}49(8.1) \\
171(28.2)\end{array}$ & $\begin{array}{c}49(4.1) \\
231(19.1)\end{array}$ & $<0.001$ & $\begin{array}{c}15(9.0) \\
42(25.1)\end{array}$ & $\begin{array}{c}34(7.6) \\
129(28.9) \\
\end{array}$ & $\begin{array}{l}0.332 \\
0.220\end{array}$ & $\begin{array}{c}25(4.2) \\
108(18.3)\end{array}$ & $\begin{array}{c}24(3.8) \\
123(19.6)\end{array}$ & $\begin{array}{l}0.255 \\
0.148\end{array}$ \\
\hline $\begin{array}{l}\text { Chronic obstructive } \\
\text { pulmonary disease }\end{array}$ & $102(16.6)$ & $188(15.5)$ & 0.475 & $8(4.8)$ & $94(21.1)$ & $<0.001$ & $53(9.0)$ & $135(21.5)$ & $<0.001$ \\
\hline Stroke & $67(10.9)$ & $116(9.5)$ & 0.646 & $17(10.2)$ & $50(11.2)$ & 0.875 & $52(8.8)$ & $64(10.2)$ & 0.609 \\
\hline $\begin{array}{c}\text { Peripheral arterial } \\
\text { disease }\end{array}$ & $104(8.5)$ & $106(17.3)$ & $<0.001$ & $22(13.2)$ & $84(18.8)$ & 0.205 & $48(8.2)$ & $56(8.9)$ & 0.395 \\
\hline $\begin{array}{c}\text { Previous myocardial } \\
\text { infarction }\end{array}$ & $412(67.2)$ & $72(5.9)$ & $<0.001$ & $117(70.1)$ & $295(66.1)$ & 0.132 & $27(4.6)$ & $45(7.7)$ & 0.065 \\
\hline $\begin{array}{l}\text { Previous coronary artery } \\
\text { revascularization }\end{array}$ & $227(37.2)$ & $55(4.5)$ & $<0.001$ & & & & & & \\
\hline - Percutaneous & $83(13.6)$ & $32(2.6)$ & & & $166(37.4)$ & 0.232 & $24(4.1)$ & $31(5.0)$ & 0.929 \\
\hline $\begin{array}{ll}- & \text { Surgical } \\
- & \text { Both }\end{array}$ & $54(8.8)$ & $15(1.2)$ & $\begin{array}{l}<0.001 \\
<0.001\end{array}$ & $\begin{array}{l}20(12.0) \\
9(5.4)\end{array}$ & $\begin{array}{l}63(14.2) \\
45(10.1)\end{array}$ & $\begin{array}{l}0.210 \\
0.199\end{array}$ & $\begin{array}{l}15(2.6) \\
7(1.2)\end{array}$ & $\begin{array}{l}17(2.7) \\
8(1.3)\end{array}$ & $\begin{array}{l}0.875 \\
0.935\end{array}$ \\
\hline Atrial fibrillation & $201(32.7)$ & $571(46.9)$ & $<0.001$ & $56(33.5)$ & $145(32.5)$ & 0.811 & $288(48.9)$ & $283(45.0)$ & 0.362 \\
\hline $\begin{array}{c}\text { Implantable cardioverter } \\
\text { defibrillator }\end{array}$ & $61(10.0)$ & $66(5.5)$ & 0.002 & $4(2.4)$ & $57(12.8)$ & $<0.001$ & $16(2.7)$ & $50(8.0)$ & $<0.001$ \\
\hline $\begin{array}{c}\text { Implantable cardioverter } \\
\text { defibrillator in patients } \\
\text { with a LVEF }<35 \%\end{array}$ & $42(15.9)$ & $42(13.3)$ & 0.382 & $2(4.8)$ & $40(95.2)$ & 0.003 & $7(16.7)$ & $35(83.3)$ & 0.126 \\
\hline $\begin{array}{c}\text { Cardiac } \\
\text { resynchronization } \\
\text { therapy }\end{array}$ & $24(3.9)$ & $27(2.2)$ & 0.130 & $3(1.8)$ & $21(4.7)$ & 0.161 & $10(1.7)$ & $17(2.7)$ & 0.485 \\
\hline Previous treatments: & & & & & & & & & \\
\hline ACEIs & $290(47.3)$ & $398(32.7)$ & $<0.001$ & $77(46.1)$ & $213(47.8)$ & 0.517 & $179(30.4)$ & 219 (34.9) & 0.128 \\
\hline ARBs & $153(24.9)$ & $291(23.9)$ & 0.373 & $50(29.9)$ & $103(23.1)$ & 0.005 & $156(26.5)$ & $135(21.5)$ & 0.093 \\
\hline Betablockers & $430(70.1)$ & $562(46.1)$ & 0.001 & $118(70.7)$ & $312(70.1)$ & 0.960 & $282(48.0)$ & $281(44.6)$ & 0.012 \\
\hline Ivabradine & $52(8.5)$ & $35(2.9)$ & $<0.001$ & $9(5.4)$ & $43(9.6)$ & 0.212 & $13(2.2)$ & $22(3.5)$ & 0.358 \\
\hline Loop diuretics & $391(63.8)$ & $687(56.5)$ & 0.004 & $104(62.3)$ & $287(64.4)$ & 0.639 & $354(60.1)$ & $333(53.0)$ & 0.043 \\
\hline $\begin{array}{l}\text { Mineralocorticoid } \\
\text { receptor antagonists }\end{array}$ & $182(29.7)$ & $255(20.9)$ & $<0.001$ & $38(22.8)$ & $144(32.3)$ & 0.004 & $124(21.1)$ & $131(20.8)$ & 0.213 \\
\hline Digoxin & $47(7.7)$ & $151(12.4)$ & 0.006 & $12(7.2)$ & $35(7.9)$ & 0.894 & $85(14.4)$ & $66(10.5)$ & 0.101 \\
\hline Oral anticoagulation & $213(34.9)$ & 507 (41.7) & 0.011 & $51(30.5)$ & $162(36.5)$ & 0.183 & $275(46.7)$ & $232(37.1)$ & 0.001 \\
\hline Aspirin & $343(56.1)$ & $294(24.2)$ & $<0.001$ & $97(58.1)$ & $246(55.4)$ & 0.552 & $128(21.7)$ & $166(26.5)$ & 0.075 \\
\hline Clopidogrel & 149 (24.4) & $48(3.9)$ & $<0.001$ & $40(24.0)$ & 109 (24.6) & 0.878 & $23(3.9)$ & $25(4.0)$ & 0.483 \\
\hline Ticagrelor & $9(1.5)$ & 0 & $<0.001$ & $5(3.0)$ & $4(0.9)$ & 0.073 & $1(0.2)$ & 0 & 0.485 \\
\hline Prasugrel & $17(2.8)$ & $1(0.1)$ & $<0.001$ & $5(3.0)$ & $12(2.7)$ & 0.846 & 0 & $1(0.2)$ & 0.250 \\
\hline
\end{tabular}

Data are shown as mean \pm standard deviation for continuous variables, and $n(\%)$ for categorical. ACEI: Angiotensin Converting Enzyme Inhibitors; ARB: Angiotensin II Receptor Blockers; GFR: Glomerular Filtration Rate; LVEF: Left Ventricular Ejection Fraction. 
In patients with ischemic etiology, men were carriers of an implantable cardioverterdefibrillator more frequently than women $(57(12.8 \%)$ vs. $4(2.4 \%), p<0.001)$, and this was also the case in patients with non-ischemic HF (50 (8.0\%) vs. $16(2.7 \%), p<0.001)$. Concerning pharmacological treatment, women with ischemic etiology more commonly received angiotensin II receptor blockers and fewer mineralocorticoid receptor antagonists compared to men.

Left ventricular ejection fraction was higher in women with ischemic etiology, compared to men ( $47 \pm 18 \%$ vs. $39 \pm 16 \%$ ) (Table 2$)$. Men, however, had greater ventricular remodeling and dilation and a wider QRS duration. Mortality during hospital admission was comparable in men and women (43 (4.0) vs. 28 (3.7)), but length of hospital stay was shorter in women $(9.8 \pm 8.5$ vs. $12.2 \pm 17.4)$. Women with ischemic HF were older, had greater comorbidity, lower left ventricular ejection fraction, and more common previous HF hospitalizations compared to women with non-ischemic HF.

Table 2. Vital signs at admission and in-hospital treatments in patients with heart failure (HF) according to etiology and sex.

\begin{tabular}{|c|c|c|c|c|c|c|c|c|c|}
\hline & $\begin{array}{c}\text { Ischemic } \\
\text { HF }(n=613)\end{array}$ & $\begin{array}{c}\text { Non- } \\
\text { Ischemic HF } \\
(n=1218)\end{array}$ & $p$ & $\begin{array}{c}\text { Women, } \\
\text { Ischemic HF } \\
(n=167)\end{array}$ & $\begin{array}{c}\text { Men, } \\
\text { Ischemic } \\
\text { HF }(n=446)\end{array}$ & $p$ & $\begin{array}{c}\text { Women, } \\
\text { Non- } \\
\text { Ischemic } \\
\text { HF }(n=589) \\
\end{array}$ & $\begin{array}{l}\text { Men, Non- } \\
\text { Ischemic } \\
\text { HF }(n=629)\end{array}$ & $p$ \\
\hline Systolic blood pressure & $134 \pm 29$ & $135 \pm 30$ & 0.8772 & $140 \pm 29$ & $132 \pm 30$ & 0.007 & $136 \pm 28$ & $133 \pm 29$ & 0.635 \\
\hline Diastolic blood pressure & $75 \pm 18$ & $76 \pm 18$ & 0.167 & $73.8 \pm 17.7$ & $75.5 \pm 18.1$ & 0.300 & $75 \pm 17$ & $77 \pm 13$ & 0.263 \\
\hline Heart rate & $86 \pm 22$ & $91 \pm 28$ & 0.002 & $85.1 \pm 23.5$ & $85.9 \pm 22.1$ & 0.690 & $91 \pm 28$ & $90 \pm 27$ & 0.756 \\
\hline Left bundle branch block & $97(16.7)$ & $179(15.3)$ & 0.006 & $24(15.1)$ & $73(17.3)$ & 0.793 & 77 (13.7) & $102(16.9)$ & 0.314 \\
\hline QRS duration (ms) & $119 \pm 33$ & $113 \pm 33$ & $<0.006$ & $110 \pm 29$ & $123 \pm 35$ & $<0.001$ & $108 \pm 31$ & $118 \pm 34$ & 0.012 \\
\hline $\begin{array}{l}\text { Left ventricular diastolic } \\
\text { diameter }(\mathrm{mm})\end{array}$ & $58 \pm 11$ & $53 \pm 11$ & $<0.001$ & $52 \pm 9$ & $60 \pm 11$ & $<0.001$ & $49 \pm 9.6$ & $57 \pm 10$ & 0.076 \\
\hline $\begin{array}{l}\text { Left ventricular ejection } \\
\text { fraction }(\%) \text { during } \\
\text { admission }\end{array}$ & $41 \pm 17$ & $48 \pm 18$ & $<0.001$ & $47 \pm 18$ & $39 \pm 16$ & $<0.001$ & $54 \pm 16$ & $43 \pm 18$ & 0.039 \\
\hline $\begin{array}{l}\text { Moderate-severe mitral } \\
\text { regurgitation }\end{array}$ & $203(33.3)$ & 401 (32.9) & 0.524 & $51(30.5)$ & $152(34.0)$ & 0.622 & $189(32.0)$ & $212(33.7)$ & 0.393 \\
\hline Sodium $(\mathrm{mEq} / \mathrm{L})$ & $138 \pm 5$ & $139 \pm 5$ & 0.236 & $138 \pm 5$ & $139 \pm 4$ & 0.070 & $139 \pm 4$ & $139 \pm 5$ & 0.051 \\
\hline Potassium (mmol/L) & $4.4 \pm 0.7$ & $4.3 \pm 0.7$ & 0.033 & $4.4 \pm 0.8$ & $4.3 \pm 0.7$ & 0.261 & $4.3 \pm 0.7$ & $4.3 \pm 0.7$ & 0.077 \\
\hline $\begin{array}{l}\text { Glomerular filtration } \\
\text { rate }(\mathrm{mL} / \mathrm{min})\end{array}$ & $60.2 \pm 29.4$ & $67.2 \pm 30.3$ & $<0.001$ & $61.8 \pm 29.9$ & $55.8 \pm 27.4$ & 0.024 & $64.1 \pm 29.4$ & $70.1 \pm 31.0$ & 0.188 \\
\hline NT-proBNP & $947 \pm 1295$ & $747 \pm 904$ & 0.001 & $990 \pm 1363$ & $929 \pm 1267$ & 0.657 & $670 \pm 858$ & $829 \pm 944$ & 0.051 \\
\hline Haemoglobin $(\mathrm{g} / \mathrm{L})$ & $12.2 \pm 20.1$ & $12.3 \pm 21.1$ & 0.011 & $11.4 \pm 14.9$ & $12.3 \pm 21.2$ & $<0.001$ & $11.8 \pm 17.8$ & $12.8 \pm 22.7$ & $<0.001$ \\
\hline $\begin{array}{c}\text { Non-invasive } \\
\text { mechanical ventilation }\end{array}$ & $36(5.9)$ & $62(5.2)$ & $<0.001$ & $6(3.7)$ & $30(6.8)$ & 0.085 & $25(4.3)$ & $37(6.0)$ & 0.226 \\
\hline $\begin{array}{c}\text { Invasive mechanical } \\
\text { ventilation }\end{array}$ & $6(1.0)$ & $8(0.7)$ & 0.075 & $1(0.6)$ & $5(1.1)$ & 0.198 & $2(0.3)$ & $6(1.0)$ & 0.210 \\
\hline Intraaortic balloon pump & $3(0.5)$ & $2(0.2)$ & $<0.001$ & $1(0.6)$ & $2(0.5)$ & 0.276 & 0 & $2(0.3)$ & 0.387 \\
\hline $\begin{array}{c}\text { Treatments at hospital } \\
\text { discharge: } \\
\text { ACEIs } \\
\text { ARBs } \\
\text { Betablockers } \\
\text { Ivabradine } \\
\text { Loop diuretics }\end{array}$ & $\begin{array}{c}339(55.3) \\
101(16.5) \\
479(80.5) \\
70(11.4) \\
512(83.5)\end{array}$ & $\begin{array}{c}579(47.6) \\
225(18.5) \\
782(66.3) \\
64(5.3) \\
1001(82.3)\end{array}$ & $\begin{array}{l}0.006 \\
0.324 \\
<0.001 \\
<0.001 \\
0.792\end{array}$ & $\begin{array}{c}90(53.9) \\
37(22.1) \\
127(77.9) \\
13(7.8) \\
143(85.6)\end{array}$ & $\begin{array}{c}249(55.8) \\
64(14.4) \\
352(81.5) \\
57(12.8) \\
369(82.7)\end{array}$ & $\begin{array}{l}0.634 \\
0.014 \\
0.354 \\
0.124 \\
0.495\end{array}$ & $\begin{array}{c}250(42.4) \\
119(20.2) \\
282(47.8) \\
22(3.7) \\
496(84.2)\end{array}$ & $\begin{array}{c}329(52.4) \\
106(16.9) \\
281(44.7) \\
42(6.7) \\
505(80.4)\end{array}$ & $\begin{array}{l}<0.001 \\
0.265 \\
0.012 \\
0.030 \\
0.180\end{array}$ \\
\hline $\begin{array}{l}\text { Mineralocorticoid } \\
\text { receptor antagonists }\end{array}$ & $269(43.9)$ & $525(43.1)$ & $<0.001$ & $62(37.1)$ & $207(46.4)$ & 0.003 & $192(33.9)$ & $181(30.2)$ & $<0.001$ \\
\hline $\begin{array}{c}\text { Digoxin } \\
\text { Oral anticoagulation } \\
\text { Aspirin } \\
\text { Clopidogrel } \\
\text { Ticagrelor } \\
\text { Prasugrel } \\
\end{array}$ & $\begin{array}{c}56(9.1) \\
266(44.7) \\
369(62.0) \\
174(29.2) \\
13(2.2) \\
9(1.5) \\
\end{array}$ & $\begin{array}{c}254(20.9) \\
696(58.9) \\
302(25.6) \\
72(6.1) \\
2(0.2) \\
1(0.1) \\
\end{array}$ & $\begin{array}{l}<0.001 \\
<0.001 \\
<0.001 \\
<0.001 \\
<0.001 \\
<0.001\end{array}$ & $\begin{array}{c}16(9.6) \\
66(40.2) \\
101(61.6) \\
48(29.3) \\
8(4.9) \\
3(1.8) \\
\end{array}$ & $\begin{array}{c}40(9.0) \\
200(46.4) \\
268(62.2) \\
126(29.2) \\
5(1.2) \\
6(1.4) \\
\end{array}$ & $\begin{array}{l}0.814 \\
0.176 \\
0.495 \\
0.994 \\
0.010 \\
0.712 \\
\end{array}$ & $\begin{array}{c}134(22.8) \\
369(64.2) \\
123(21.4) \\
27(4.7) \\
0 \\
0 \\
\end{array}$ & $\begin{array}{c}120(19.1) \\
327(54.1) \\
179(29.6) \\
45(7.4) \\
2(0.3) \\
1(0.2) \\
\end{array}$ & $\begin{array}{l}0.287 \\
<0.001 \\
0.001 \\
0.018 \\
0.035 \\
0.069 \\
\end{array}$ \\
\hline
\end{tabular}


Table 2. Cont.

\begin{tabular}{|c|c|c|c|c|c|c|c|c|c|}
\hline & $\begin{array}{l}\text { Ischemic } \\
\text { HF }(n=613)\end{array}$ & $\begin{array}{c}\text { Non- } \\
\text { Ischemic HF } \\
(n=1218)\end{array}$ & $p$ & $\begin{array}{l}\text { Women, } \\
\text { Ischemic HF } \\
(n=167)\end{array}$ & $\begin{array}{c}\text { Men, } \\
\text { Ischemic } \\
\text { HF }(n=446)\end{array}$ & $p$ & $\begin{array}{c}\text { Women, } \\
\text { Non- } \\
\text { Ischemic } \\
\text { HF }(n=589)\end{array}$ & $\begin{array}{l}\text { Men, Non- } \\
\text { Ischemic } \\
\text { HF }(n=629)\end{array}$ & $p$ \\
\hline $\begin{array}{l}\text { Percutaneous coronary } \\
\text { artery revascularization } \\
\text { during admission }\end{array}$ & $66(11.0)$ & $35(2.9)$ & $<0.001$ & $17(10.4)$ & 49 (11.2) & 0.651 & $12(2.1)$ & $23(3.7)$ & 0.171 \\
\hline $\begin{array}{l}\text { Surgical coronary artery } \\
\text { revascularization during } \\
\text { admission }\end{array}$ & $6(0.5)$ & $12(2.0)$ & 0.013 & $1(0.6)$ & $11(2.5)$ & 0.172 & $3(0.5)$ & $3(0.5)$ & 0.726 \\
\hline $\begin{array}{l}\text { Death during hospital } \\
\text { admission }\end{array}$ & $45(3.7)$ & $26(4.3)$ & 0.851 & $8(4.8)$ & $18(4.0)$ & 0.202 & $20(3.4)$ & $25(4.0)$ & 0.703 \\
\hline $\begin{array}{l}\text { Length of hospital stay } \\
\text { (days) }\end{array}$ & $11.1 \pm 9.5$ & $11.3 \pm 16.4$ & 0.837 & $8.8 \pm 5.5$ & $12.0 \pm 10.5$ & 0.002 & $10.1 \pm 9.1$ & $12.4 \pm 21.0$ & $<0.001$ \\
\hline
\end{tabular}

Data are shown as mean \pm standard deviation for continuous variables, and $n(\%)$ for categorical. ACEI: Angiotensin Converting Enzyme Inhibitors; ARB: Angiotensin II Receptor Blockers; NT-proBNP: N-terminal (NT)-pro hormone BNP.

Unadjusted analysis of mortality and hospital readmissions showed similar outcomes during follow-up among men and women with ischemic etiology of HF (Table 3). However, women with non-ischemic etiology tended to present fewer hospital readmissions at 12 months. In adjusted Cox regression analysis, ischemic etiology was not associated with an increased mortality at 12 months in women (HR 1.52, 95\% CI 0.97-2.33, $p=0.052$ ) or men (HR 1.28, 95\% CI 0.911-1.79, $p=0.155$ ) (Figure 1). Women received a heart transplant less often compared to men in univariate comparisons (5 (0.7\%) vs. $31(2.9 \%), p=0.05)$. After a multivariate analysis adjusting for age and comorbidities, we did not find sex-related differences in heart transplants at 12 months (HR 2.53, 95\% CI 0.87-7.39; $p=0.09$ ). The only factor associated with a lower indication of heart transplants was older age (HR 0.92, 95\% CI 0.89-0.94; $p<0.001)$.

Table 3. Events during follow-up in patients admitted with heart failure (HF) according to etiology and sex.

\begin{tabular}{|c|c|c|c|c|c|c|}
\hline & $\begin{array}{c}\text { Women, } \\
\text { Ischemic HF } \\
(n=167)\end{array}$ & $\begin{array}{c}\text { Men, } \\
\text { Ischemic HF } \\
(n=446)\end{array}$ & $p$ & $\begin{array}{c}\text { Women, } \\
\text { Non-Ischemic HF } \\
(n=589)\end{array}$ & $\begin{array}{c}\text { Men, } \\
\text { Non-Ischemic HF } \\
(n=629)\end{array}$ & $p$ \\
\hline $\begin{array}{l}\text { Hospital readmissions } \\
\text { due to } \mathrm{HF} \text { at } 12 \text { months }\end{array}$ & $57(34.1)$ & $146(32.7)$ & 0.773 & $181(30.7)$ & $159(25.3)$ & 0.034 \\
\hline $\begin{array}{l}\text { All-cause mortality at } \\
12 \text { months }\end{array}$ & $42(25.2)$ & $102(22.9)$ & 0.593 & $111(18.9)$ & $120(19.1)$ & 0.918 \\
\hline $\begin{array}{l}\text { Heart transplant at } \\
12 \text { months }\end{array}$ & $1(0.6)$ & $13(2.9)$ & 0.050 & $4(0.7)$ & $18(2.9)$ & 0.004 \\
\hline $\begin{array}{l}\text { Sudden cardiac death at } \\
12 \text { months }\end{array}$ & $4(2.4)$ & $18(4.0)$ & 0.331 & $10(1.7)$ & $23(3.7)$ & 0.035 \\
\hline $\begin{array}{c}\text { Death due to refractory } \\
\text { heart failure }\end{array}$ & 19 (11.4) & 52 (11.7) & 0.923 & $59(10.0)$ & $44(7.0)$ & 0.058 \\
\hline $\begin{array}{l}\text { Death due to non-cardiac } \\
\text { causes }\end{array}$ & $13(7.8)$ & $24(5.4)$ & 0.259 & $25(4.2)$ & $36(5.7)$ & 0.293 \\
\hline
\end{tabular}

Data are shown as mean \pm standard deviation for continuous variables, and $n(\%)$ for categorical.

Ischemic etiology was not an independent predictor of mortality (HR 1.21, 95\% CI 0.96-1.53, $p=0.11$ ) (Table 4), and this was true for women (HR 1.51, 95\% CI 0.98-2.32; $p=0.61$ ) and men (HR 1.14, 95\% CI 0.81-1.61; $p=0.46$ ), interaction $p$-value: 0.067 . Ischemic etiology was also not associated with the combined endpoint of mortality/readmissions. In patients with ischemic HF, diabetes was associated with an increased risk of mortality/readmissions in women and previous HF admissions and chronic obstructive pulmonary disease in men. 


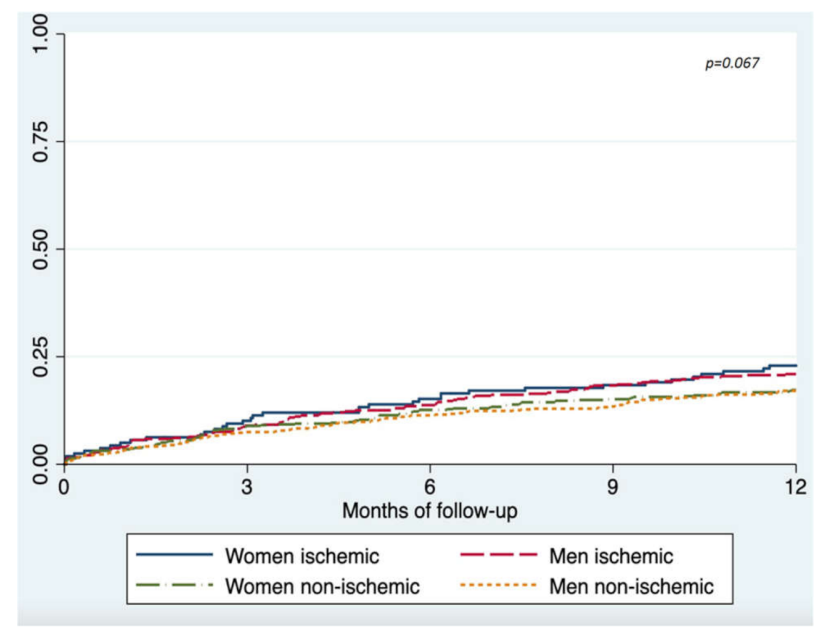

Figure 1. Show Kaplan-Meier survival curves of mortality at 12 months according to HF etiology, in men and women.

Table 4. Independent predictors of mortality, and mortality/readmissions at 12 months in patients admitted with heart failure (HF) according to etiology and sex.

\begin{tabular}{|c|c|c|}
\hline \multicolumn{3}{|c|}{ Women, Ischemic HF } \\
\hline 12-Month Mortality & HR (95\% CI) & $p$ \\
\hline - $\quad$ ACEIs/ARBs & $0.58(0.24-0.97)$ & 0.041 \\
\hline 12-Month Mortality/Readmissions & HR $(95 \%$ CI) & $p$ \\
\hline Diabetes mellitus & $2.23(1.05-4.47)$ & 0.035 \\
\hline \multicolumn{3}{|c|}{ Men, Ischemic HF } \\
\hline 12-Month Mortality & HR $(95 \%$ CI) & $p$ \\
\hline$-\quad$ Age & $1.03(1.01-1.05)$ & 0.010 \\
\hline - $\quad$ ACEIs/ARBs & $0.58(0.33-0.79)$ & 0.002 \\
\hline 12-Month Mortality/Readmissions & $\operatorname{HR}(95 \% \mathrm{CI})$ & $p$ \\
\hline - $\quad$ Previous HF admissions & $1.15(1.02-1.29)$ & 0.022 \\
\hline $\begin{array}{l}\text { - Chronic obstructive pulmonary } \\
\text { disease }\end{array}$ & $1.20(1.02-1.41)$ & 0.026 \\
\hline \multicolumn{3}{|c|}{ Women, Non-Ischemic HF } \\
\hline 12-Month Mortality & HR $(95 \%$ CI) & $p$ \\
\hline Age & $1.04(1.14-1.06)$ & 0.002 \\
\hline - $\quad$ Diabetes mellitus & $1.34(1.13-1.58)$ & 0.001 \\
\hline - $\quad$ Chronic kidney disease & $1.28(1.07-1.52)$ & 0.008 \\
\hline - $\quad$ ACEIs/ARBs & $0.46(0.31-0.69)$ & $<0.001$ \\
\hline
\end{tabular}


Table 4. Cont.

\begin{tabular}{|c|c|c|}
\hline \multicolumn{3}{|c|}{ Women, Non-Ischemic HF } \\
\hline 12-Month Mortality/Readmissions & HR $(95 \%$ CI $)$ & $p$ \\
\hline - $\quad$ Previous HF admissions & $1.25(1.14-1.38)$ & $<0.001$ \\
\hline \multicolumn{3}{|c|}{ Men, Non-Ischemic HF } \\
\hline 12-Month Mortality & HR $(95 \%$ CI $)$ & $p$ \\
\hline Age & $1.05(1.03-1.67)$ & $<0.001$ \\
\hline - $\quad$ Previous HF admissions & $1.88(1.03-1.67)$ & 0.004 \\
\hline - $\quad$ Chronic kidney disease & $1.22(1.08-1.39)$ & 0.002 \\
\hline - $\quad$ ACEIs/ARBs & $0.61(0.40-0.91)$ & 0.015 \\
\hline 12-Month Mortality/Readmissions & HR $(95 \%$ CI) & $p$ \\
\hline$-\quad$ Age & $1.02(1.01-1.03)$ & 0.021 \\
\hline - $\quad$ Previous HF admissions & $1.28(1.17-1.39)$ & $<0.001$ \\
\hline - $\quad$ ACEIs/ARBs & $0.69(0.50-0.97)$ & 0.033 \\
\hline
\end{tabular}

ACEI: Angiotensin Converting Enzyme Inhibitors; ARB: Angiotensin II Receptor Blockers; CI: Confidence Interval; HF: Heart Failure; HR: Hazard Ratio.

\section{Discussion}

We have found that, among patients admitted with HF, ischemic etiology is present in a fifth of women and about $40 \%$ of men. Ischemic etiology was not associated with an increased risk of adverse events.

\subsection{Comorbidities and Cardiovascular Risk Factors}

Women are underrepresented in clinical trials, and patients from clinical trials are highly selected. Our sample is more representative of real-world patients. Previous studies that have analyzed HF's presentation in women have shown frequent advanced age and comorbidity [21-24]. In contrast to men, the most common form of HF in women is that with preserved left ventricular function [22]. Our results were consistent with previous experiences, as women had higher left ventricular ejection fraction and older age $[8,21,25]$. Regarding comorbidity and cardiovascular risk factors, women with ischemic etiology frequently had diabetes (69\%), but the rate of hypertension was comparable to men (Supplementary Materials). It is possible that high blood pressure plays a less prominent role in the development of ischemic heart disease in women, which would be more related to other cardiovascular risk factors, such as diabetes [23,26]. HF risk is higher in women with diabetes than in men with diabetes [23,26-28]. Analyzing the characteristics of women with ischemic etiology, we have observed that the clinical profile of these patients is more similar to men with ischemic HF than to women with HF of non-ischemic etiology, suggesting that several differences associated with sex might be, in fact, related to ischemic etiology.

\subsection{Heart Failure Therapies}

The use of a implantable cardioverter-defibrillator was higher in men than in women. This might be related, in part, to the fact that men more frequently had a left ventricular 
ejection fraction $<35 \%[18,25]$. However, it has also previously been stated that women with $\mathrm{HF}$ and a reduced left ventricular ejection fraction are less likely to be referred for cardioverter-defibrillator implantation [29]. In fact, in the specific group of patients with ischemic etiology and reduced left ventricular ejection fraction, the proportion of women with a defibrillator was also lower than men. In our study, the incidence of sudden cardiac death during follow-up was similar between men and women with ischemic HF.

Traditionally, women with HF have been under-treated compared to men. This difference has been attributed to women's more unfavorable clinical profile, with older age and common comorbidities [30]. However, in the subgroup of women with ischemic etiology, we only found significant differences in the prescription of mineralocorticoid receptor antagonists. The lower prescription of these drugs in the cohort of our study may also be explained by the lack of indication due to the higher left ventricular ejection fraction in women [25].

\subsection{Mortality and Hospital Readmissions during Follow-Up}

We did not find significant sex differences in hospital mortality and mortality/readmissions during follow-up among patients with ischemic HF.

In some previous studies, women have shown better-adjusted survival rates than in men [16,29,31-33], a finding which is more marked in patients with non-ischemic HF $[23,34,35]$. In our registry patients, the survival advantage traditionally attributed to women with non-ischemic heart failure may have been attenuated by a more advanced disease, with more years since the diagnosis.

With regard to hospitalization for heart failure, previous experiences have described that the male sex is associated with a higher probability of readmission $[36,37]$ and the combined endpoint of mortality/readmissions [36]. The attenuation of these sex-related differences in prognosis could be explained by HF's etiology and the negative impact of coronary heart disease in women. Indeed, women who present with an acute myocardial infarction have an increased risk of developing heart failure during admission and followup, compared to men [11,12]. Mental/psychological stress was not assessed in this study. Future studies should analyze the impact of psychosocial dimension on the prognosis of patients with HF according to etiology, and sex differences.

Other variables associated with worse outcomes were comorbidities, especially diabetes mellitus or chronic obstructive pulmonary disease in women, and previous hospitalizations in men with ischemic etiology. Treatment with ACEIs/ARBs was a factor associated with a lower risk of death and readmissions, regardless of sex and the etiology of heart failure.

Women tend to receive heart transplants less often than men [38]. In the univariate analysis of our study, heart transplants were performed less frequently in women. However, after multivariate adjustment, there were no differences between men and women with ischemic or non-ischemic etiology of HF. Advanced age was the only factor associated with a lower probability of receiving a heart transplant, and therefore, after statistical adjustment, the indication for transplant was similar in both sexes.

Our study has some limitations. The follow-up phase was 12 months, and a longer follow-up period may have shown significant differences in outcomes according to HF etiology and sex. The dosage of disease-modifying drugs was not recorded, and we do not have information about patients who presented appropriate defibrillator shocks during follow-up.

\section{Conclusions}

Compared to patients with non-ischemic etiology, patients with ischemic HF have more comorbidities. Ischemic etiology was not associated with mortality in HF patients. In ischemic HF, the variables associated with a poor prognosis were diabetes in women, and previous readmissions and chronic obstructive pulmonary disease in men. Additional 
studies are required to determine the specific impact of other etiologies on the prognosis of HF.

Supplementary Materials: The following are available online at https://www.mdpi.com/article/10 $.3390 /$ jcm10081713/s1, Table S1: Basal demographic and clinical characteristics of patients with HF according to sex and etiology.

Author Contributions: Conceptualization: M.M.-S. and L.V.; Methodology, M.M.-S. and L.V.; Software, M.M.-S. and L.V.; Validation, J.G., L.M.D., R.V.-G., J.R.G.-J., J.S., D.P.-F., R.B., F.W., J.D., F.F.-A. and M.M.-S.; Formal Analysis, M.M.-S., and L.V.; Investigation, L.M.D., R.V.-G., J.R.G.-J., J.S., D.P.-F., R.B., F.W., J.D., F.F.-A. and M.M.-S.; Resources, R.V.-G., J.R.G.-J., J.S., D.P.-F., R.B., F.W., J.D., F.F.A. and M.M.-S.; Data Curation, R.V.-G., J.R.G.-J., J.S., D.P.-F., R.B., F.W., J.D., F.F.-A. and M.M.-S.; Writing-Original Draft Preparation, L.V. and M.M.-S.; Writing-Review and Editing, L.V. and M.M.-S.; Visualization, R.V.-G., J.R.G.-J., J.S., D.P.-F., R.B., F.W., J.D., F.F.-A. and M.M.-S.; Supervision, R.V.-G., J.R.G.-J., J.S., D.P.-F., R.B., F.W., J.D., F.F.-A. and M.M.-S.; Project Administration, R.V.-G., J.R.G.-J., J.S., D.P.-F., R.B., F.W., J.D., F.F.-A. and M.M.-S.; Funding Acquisition, R.V.-G., J.R.G.-J., J.S., D.P.-F., R.B., F.W., J.D., F.F.-A. and M.M.-S. All authors have read and agreed to the published version of the manuscript.

Funding: This research received no external funding.

Institutional Review Board Statement: This study accomplishes with the Declaration of Helsinki and was approved by the Ethics Committee of the recruiting hospitals (9/12/2013 CEIC: 57/2013; 19/09/2013 CEIC: 13/2013).

Informed Consent Statement: All patients provided written informed consent.

Data Availability Statement: Data will be provided by request.

Conflicts of Interest: The authors declare no conflict of interest.

\section{References}

1. Khan, M.A.; Hashim, M.J.; Mustafa, H.; Baniyas, M.Y.; Al Suwaidi, S.K.B.M.; AlKatheeri, R.; Alblooshi, F.M.K.; Almatrooshi, M.E.A.H.; Alzaabi, M.E.H.; Al Darmaki, R.S.; et al. Global epidemiology of ischemic heart disease: Results from the Global Burden of Disease Study. Cureus 2020, 12, e9349. [CrossRef] [PubMed]

2. Velagaleti, R.S.; Vasan, R.S. Heart failure in the twenty-first century: Is it a coronary artery disease or hypertension problem? Cardiol. Clin. 2007, 25, 487-495. [CrossRef] [PubMed]

3. Elgendy, I.Y.; Mahtta, D.; Pepine, C.J. Medical therapy for heart failure caused by ischemic heart disease. Circ. Res. 2019, 124, 1520-1535. [CrossRef] [PubMed]

4. Qureshi, W.T.; Zhang, Z.-M.; Chang, P.P.; Rosamond, W.D.; Kitzman, D.W.; Wagenknecht, L.E.; Soliman, E.Z. Silent Myocardial Infarction and Long-Term Risk of Heart Failure: The ARIC Study. J. Am. Coll. Cardiol. 2018, 71, 1-8. [CrossRef]

5. Pecini, R.; Møller, D.V.; Torp-Pedersen, C.; Hassager, C.; Køber, L. Heart failure etiology impacts survival of patients with heart failure. Int. J. Cardiol. 2011, 149, 211-215. [CrossRef]

6. Balmforth, C.; Simpson, J.; Shen, L.; Jhund, P.S.; Lefkowitz, M.; Rizkala, A.R.; Rouleau, J.L.; Shi, V.; Solomon, S.D.; Swedberg, K.; et al. Outcomes and effect of treatment according to etiology in HFrEF: An analysis of PARADIGM-HF. JACC Heart Fail. 2019, 7, 457-465. [CrossRef] [PubMed]

7. Mentzer, G.; Hsich, E.M. Heart Failure with Reduced Ejection Fraction in Women: Epidemiology, Outcomes, and Treatment. Heart Fail. Clin. 2019, 15, 19-27. [CrossRef]

8. Tibrewala, A.; Yancy, C.W. Heart failure with preserved ejection fraction in women. Heart Fail. Clin. 2019, 15, 9-18. [CrossRef]

9. Postigo, A.; Martínez-Sellés, M. Sex influence on heart failure prognosis. Front. Cardiovasc. Med. 2020, 7, 356. [CrossRef]

10. Heras, M. Ischemic heart disease in women: Clinical presentation, non-invasive testing and management of acute coronary syndromes. Rev. Esp. Cardiol. Engl. Ed. 2006, 59, 371-381. [CrossRef]

11. Lewis, E.F.; Velazquez, E.J.; Solomon, S.D.; Hellkamp, A.S.; McMurray, J.J.V.; Mathias, J.; Rouleau, J.-L.; Maggioni, A.P.; Swedberg, K.; Kober, L.; et al. Predictors of the first heart failure hospitalization in patients who are stable survivors of myocardial infarction complicated by pulmonary congestion and/or left ventricular dysfunction: A VALIANT study. Eur. Heart J. 2008, 29, 748-756. [CrossRef]

12. French, J.K.; Armstrong, P.W.; Cohen, E.; Kleiman, N.S.; O'Connor, C.M.; Hellkamp, A.S.; Stebbins, A.; Holmes, D.R.; Hochman, J.S.; Granger, C.B.; et al. Cardiogenic shock and heart failure post-percutaneous coronary intervention in ST-elevation myocardial infarction: Observations from "Assessment of Pexelizumab in Acute Myocardial Infarction". Am. Heart J. 2011, 162, 89-97. [CrossRef] 
13. Mosca, L.; Manson, J.E.; Sutherland, S.E.; Langer, R.D.; Manolio, T.; Barrett-Connor, E. Cardiovascular Disease in Women. Circulation 1997, 96, 2468-2482. [CrossRef]

14. Vicent, L.; Ariza-Solé, A.; Alegre, O.; Sanchís, J.; López-Palop, R.; Formiga, F.; González-Salvado, V.; Bueno, H.; Vidán, M.T.; Díez-Villanueva, P.; et al. Octogenarian women with acute coronary syndrome present frailty and readmissions more frequently than men. Eur. Heart J. Acute Cardiovasc. Care 2019, 8, 252-263. [CrossRef]

15. Melloni, C.; Berger, J.S.; Wang, T.Y.; Gunes, F.; Stebbins, A.; Pieper, K.S.; Dolor, R.J.; Douglas, P.S.; Mark, D.B.; Newby, L.K. Representation of women in randomized clinical trials of cardiovascular disease prevention. Circ. Cardiovasc. Qual. Outcomes 2010, 3, 135-142. [CrossRef]

16. Martínez-Sellés, M.; Díez-Villanueva, P.; Álvarez Garcia, J.; Ferrero-Gregori, A.; Vives-Borrás, M.; Worner, F.; Bardají, A.; Delgado, J.F.; Vázquez, R.; González-Juanatey, J.R.; et al. Influence of sex and pregnancy on survival in patients admitted with heart failure: Data from a prospective multicenter registry. Clin. Cardiol. 2018, 41, 924-930. [CrossRef]

17. Vicent, L.; Cinca, J.; Vazquez-García, R.; Gonzalez-Juanatey, J.R.; Rivera, M.; Segovia, J.; Pascual-Figal, D.; Bover, R.; Worner, F.; Delgado-Jiménez, J.; et al. Discharge treatment with angiotensin-converting enzyme inhibitor/angiotensin receptor blocker after a heart failure hospitalisation is associated with a better prognosis irrespective of left ventricular ejection fraction. Intern. Med. J. 2019, 49, 1505-1513. [CrossRef]

18. McMurray, J.J.V.; Adamopoulos, S.; Anker, S.D.; Auricchio, A.; Böhm, M.; Dickstein, K.; Falk, V.; Filippatos, G.; Fonseca, C.; Gomez-Sanchez, M.A.; et al. ESC Guidelines for the diagnosis and treatment of acute and chronic heart failure 2012: The Task Force for the Diagnosis and Treatment of Acute and Chronic Heart Failure 2012 of the European Society of Cardiology. Developed in collaboration with the Heart Failure Association (HFA) of the ESC. Eur. Heart J. 2012, 33, 1787-1847. [CrossRef]

19. Lala, A.; Desai, A.S. The role of coronary artery disease in heart failure. Heart Fail. Clin. 2014, 10, 353-365. [CrossRef]

20. Felker, G.M.; Shaw, L.K.; O'Connor, C.M. A standardized definition of ischemic cardiomyopathy for use in clinical research. J. Am. Coll. Cardiol. 2002, 39, 210-218. [CrossRef]

21. Bozkurt, B.; Khalaf, S. Heart failure in women. Methodist DeBakey Cardiovasc. J. 2017, 13, 216-223. [CrossRef] [PubMed]

22. Hogg, K.; Swedberg, K.; McMurray, J. Heart failure with preserved left ventricular systolic function; epidemiology, clinical characteristics, and prognosis. J. Am. Coll. Cardiol. 2004, 43, 317-327. [CrossRef]

23. Martínez-Sellés, M.; Doughty, R.N.; Poppe, K.; Whalley, G.A.; Earle, N.; Tribouilloy, C.; McMurray, J.J.V.; Swedberg, K.; Køber, L.; Berry, C.; et al. Gender and survival in patients with heart failure: Interactions with diabetes and aetiology. Results from the MAGGIC individual patient meta-analysis. Eur. J. Heart Fail. 2012, 14, 473-479. [CrossRef] [PubMed]

24. Elgendy, I.Y.; Pepine, C.J. Heart failure with preserved ejection fraction: Is ischemia due to coronary microvascular dysfunction a mechanistic factor? Am. J. Med. 2019, 132, 692-697. [CrossRef] [PubMed]

25. Ponikowski, P.; Voors, A.A.; Anker, S.D.; Bueno, H.; Cleland, J.G.F.; Coats, A.J.S.; Falk, V.; González-Juanatey, J.R.; Harjola, V.-P.; Jankowska, E.A.; et al. 2016 ESC Guidelines for the diagnosis and treatment of acute and chronic heart failure: The Task Force for the diagnosis and treatment of acute and chronic heart failure of the European Society of Cardiology (ESC)Developed with the special contribution of the Heart Failure Association (HFA) of the ESC. Eur. Heart J. 2016, 37, 2129-2200. [CrossRef] [PubMed]

26. Ohkuma, T.; Komorita, Y.; Peters, S.A.E.; Woodward, M. Diabetes as a risk factor for heart failure in women and men: A systematic review and meta-analysis of 47 cohorts including 12 million individuals. Diabetologia 2019, 62, 1550-1560. [CrossRef] [PubMed]

27. Kodama, S.; Fujihara, K.; Horikawa, C.; Sato, T.; Iwanaga, M.; Yamada, T.; Kato, K.; Watanabe, K.; Shimano, H.; Izumi, T.; et al Diabetes mellitus and risk of new-onset and recurrent heart failure: A systematic review and meta-analysis. ESC Heart Fail. 2020, 7, 2146-2174. [CrossRef]

28. Huxley, R.; Barzi, F.; Woodward, M. Excess risk of fatal coronary heart disease associated with diabetes in men and women: Meta-analysis of 37 prospective cohort studies. BMJ 2006, 332, 73-78. [CrossRef]

29. Gillis, A.M. Atrial fibrillation and ventricular arrhythmias: Sex differences in electrophysiology, epidemiology, clinical presentation, and clinical outcomes. Circulation 2017, 135, 593-608. [CrossRef]

30. Schall, M.B.; Flannery, J. Undertreatment of women with heart failure: A reversible outcome on hospital readmission. Lippincotts Case Manag. Manag. Process. Patient Care 2004, 9, 250-253. [CrossRef]

31. Deswal, A.; Bozkurt, B. Comparison of morbidity in women versus men with heart failure and preserved ejection fraction. Am. J. Cardiol. 2006, 97, 1228-1231. [CrossRef]

32. Regitz-Zagrosek, V.; Oertelt-Prigione, S.; Prescott, E.; Franconi, F.; Gerdts, E.; Foryst-Ludwig, A.; Maas, A.H.E.M.; Kautzky-Willer, A.; Knappe-Wegner, D.; Kintscher, U.; et al. Gender in cardiovascular diseases: Impact on clinical manifestations, management, and outcomes. Eur. Heart J. 2016, 37, 24-34. [CrossRef]

33. Martínez-Sellés, M.; García Robles, J.A.; Prieto, L.; Domínguez Muñoa, M.; Frades, E.; Díaz-Castro, O.; Almendral, J. Systolic dysfunction is a predictor of long term mortality in men but not in women with heart failure. Eur. Heart J. 2003, 24, $2046-2053$. [CrossRef]

34. Ghali, J.K.; Krause-Steinrauf, H.J.; Adams, K.F.; Khan, S.S.; Rosenberg, Y.D.; Yancy, C.W.; Young, J.B.; Goldman, S.; Peberdy, M.A.; Lindenfeld, J. Gender differences in advanced heart failure: Insights from the BEST study. J. Am. Coll. Cardiol. 2003, 42, $2128-2134$. [CrossRef]

35. Dunlay, S.M.; Roger, V.L. Gender differences in the pathophysiology, clinical presentation, and outcomes of ischemic heart failure. Curr. Heart Fail. Rep. 2012, 9, 267-276. [CrossRef] 
36. Ieva, F.; Jackson, C.H.; Sharples, L.D. Multi-state modelling of repeated hospitalisation and death in patients with heart failure: The use of large administrative databases in clinical epidemiology. Stat. Methods Med. Res. 2017, 26, 1350-1372. [CrossRef]

37. Nieminen, M.S.; Harjola, V.-P.; Hochadel, M.; Drexler, H.; Komajda, M.; Brutsaert, D.; Dickstein, K.; Ponikowski, P.; Tavazzi, L.; Follath, F; et al. Gender related differences in patients presenting with acute heart failure. Results from EuroHeart Failure Survey II. Eur. J. Heart Fail. 2008, 10, 140-148. [CrossRef]

38. Lund, L.H.; Khush, K.K.; Cherikh, W.S.; Goldfarb, S.; Kucheryavaya, A.Y.; Levvey, B.J.; Meiser, B.; Rossano, J.W.; Chambers, D.C.; Yusen, R.D.; et al. The Registry of the International Society for Heart and Lung Transplantation: Thirty-fourth adult heart transplantation report-2017; focus theme: Allograft ischemic time. J. Heart Lung Transplant. Off. Publ. Int. Soc. Heart Transplant. 2017, 36, 1037-1046. [CrossRef] 